\title{
Odd unimodular lattices of minimum 4
}

\author{
by \\ Christine Bachoc (Talence), Gabriele Nebe (Ulm) \\ and Boris Venkov (St Petersburg)
}

1. Introduction. Unimodular lattices have focused interest for a long time. One of the most fascinating properties of a unimodular lattice $\Lambda$ is that its theta series

$$
\theta_{\Lambda}(\tau):=\sum_{x \in \Lambda} q^{(x \cdot x)},
$$

where $\tau \in \mathfrak{h}$, the complex upper half plane, and $q:=e^{\pi i \tau}$, has an invariance property under the transformation $\tau \mapsto-1 / \tau$. If the lattice is moreover even, then its theta series is invariant under the action of the full modular group $\operatorname{SL}(2, \mathbb{Z})$, which leads to the upper bound for the minimum of the lattice:

$$
\min (\Lambda) \leq 2[n / 24]+2
$$

where $n$ is the dimension of the lattice. The first case where this bound is not known to be tight is $n=72$.

It is much more difficult to obtain a good bound for the minimum of an odd unimodular lattice, although these lattices are expected to be not so good as the even ones, as is observed in small dimensions. The theta series of such a lattice is only invariant under the congruence subgroup $\Gamma_{0}(4)$ and the bound derived from this invariance is $\min (\Lambda) \leq 2[n / 8]+2$, which is not sharp. Only recently, E. Rains and N. J. A. Sloane have proved that (2) holds also for the odd lattices, apart from the exceptional case $n=23$ ([6]). Their proof makes use of the theta series of the shadow of the lattice. J. H. Conway and N. J. A. Sloane have given in [3] the exact bound for the minimum of a unimodular lattice of dimension $n \leq 33$. In particular they show that there cannot exist a minimum 4 lattice of dimension 33 . We extend here this result:

2000 Mathematics Subject Classification: 11H06, $11 \mathrm{H} 50$.

Key words and phrases: lattice, unimodular, theta series.

The third author thanks the Laboratoire A2X, Université Bordeaux I, and the Heidelberg-Mannheim research group Arithmetik for financial support. 
THEOREM 1. There is no unimodular lattice of minimum 4 and dimension 34 or 35 .

Unimodular lattices of minimum 4 are known in dimensions 36, 38, 39, 40 (see [5]); the only remaining case is $n=37$.

Seeking for a contradiction, we shall first compute the theta series of a putative lattice $\Lambda$ of minimum 4 and of its shadow $S$. Then we shall compute the number of vectors of the lattice with prescribed scalar product with a fixed minimal vector of $S$. This amounts to the computation of certain coefficients of some Jacobi theta series associated to the lattice and therefore we shall make use of spherical theta series $\theta_{\Lambda, P}$ where $P$ is a harmonic polynomial.

The paper is organized as follows: Section 2 recalls results on the shadows of unimodular lattices. Section 3 introduces a 40-dimensional even unimodular lattice associated to $\Lambda$. Section 4 introduces theta series with spherical coefficients and Section 5 derives equations on the above mentioned numbers. Section 6 ends the proof of Theorem 1 .

2. Shadows. Let $\Lambda$ be an odd unimodular lattice. The shadow $S$ of $\Lambda$ is $S:=\left(\Lambda_{0}\right)^{*} \backslash \Lambda$, where $\Lambda_{0}$ denotes the even sublattice of $\Lambda$. The theta series of $\Lambda$ has the following expression:

$$
\theta_{\Lambda}(\tau)=\sum_{j=0}^{[n / 8]} a_{j} \Delta_{8}(q)^{j} \theta_{3}(q)^{n-8 j}
$$

and the theta series of the shadow $S$ is

$$
\theta_{S}(\tau)=\sum_{j=0}^{[n / 8]} \frac{(-1)^{j}}{16^{j}} a_{j} \theta_{4}\left(q^{2}\right)^{8 j} \theta_{2}(q)^{n-8 j},
$$

where $q:=e^{\pi i \tau}, \Delta_{8}(q)=q \prod_{m=1}^{\infty}\left(1-q^{2 m-1}\right)^{8}\left(1-q^{4 m}\right)^{8}$, and $\theta_{2}, \theta_{3}, \theta_{4}$ are the usual Jacobi theta series (see [2, Chap. 4, §4]).

For the rest of the paper, $\Lambda$ is an odd unimodular lattice of minimum 4 and dimension $n \geq 34$. We denote by $m$ the minimum of the shadow $S$ of $\Lambda$ and by $s_{m}$ the number of vectors $s \in S$ with $s \cdot s=m$.

If $A$ is any set of vectors, $A_{r}$ is the set of vectors $a \in A$ with $a \cdot a=r$.

We start with the computation of the theta series. For $n=34,35$, the condition that the minimum of the lattice is at least 4 determines the values of $a_{1}, a_{2}, a_{3}$. If $x \in S$, then $2 x \in \Lambda$ so the minimum of $S$ must be at least 2 . This condition forces $a_{4}=0$. We find the following theta series:

For $n=34$,

$$
\theta_{\Lambda}=1+60180 q^{4}+\ldots, \quad \theta_{S}=204 q^{5 / 2}+758200 q^{9 / 2}+\ldots
$$


For $n=35$,

$$
\theta_{\Lambda}=1+51030 q^{4}+\ldots, \quad \theta_{S}=420 q^{11 / 4}+1704780 q^{19 / 4}+\ldots
$$

We now fix a vector $s \in S_{m}$. If $s^{\prime}$ is another minimal vector in $S$, not equal to $\pm s$, then

$$
s \cdot s^{\prime} \equiv s \cdot s \bmod \frac{1}{2} \mathbb{Z} \quad \text { because } \quad s-s^{\prime} \in \Lambda,
$$

and

$$
\left|s \cdot s^{\prime}\right| \leq m-2 \quad \text { because } \quad\left(s \pm s^{\prime}\right)^{2} \geq 4 .
$$

Hence

$$
s \cdot s^{\prime} \in\{ \pm(m-[2 m] / 2), \ldots, \pm(m-5 / 2), \pm(m-2)\}
$$

We get

$$
s \cdot s^{\prime} \in \begin{cases}\{0, \pm 1 / 2\} & \text { for } n=34 \\ \{ \pm 1 / 4, \pm 3 / 4\} & \text { for } n=35\end{cases}
$$

Let $x \in \Lambda_{4}$. Since $(s \pm x)^{2} \geq m$ and $s \cdot x$ is an integer, $s \cdot x \in\{0, \pm 1, \pm 2\}$. We define

$$
\begin{aligned}
p_{i}(s) & :=\operatorname{card}\left\{x \in \Lambda \mid x^{2}=4, s \cdot x= \pm i\right\}, \quad i=0,1,2, \\
m_{i}(s): & =\operatorname{card}\left\{s^{\prime} \in S \mid s^{\prime 2}=m, s \cdot s^{\prime}= \pm i\right\}, \\
& i=m-[2 m] / 2, \ldots, m-2,
\end{aligned}
$$

Our first task is to compute these numbers for $n=34,35$. It will turn out that they do not depend on the choice of $s$. In order to determine them, we need five equations; two trivial equations come from the knowledge of $\theta_{\Lambda}$ :

$$
\begin{aligned}
\sum p_{i}(s) & =\operatorname{card}\left(\Lambda_{4}\right), \\
\sum m_{i}(s) & =\operatorname{card}\left(S_{m}\right)-2 .
\end{aligned}
$$

Some more equations will come from theta series with spherical coefficients. In order to avoid the use of half integral weight modular forms we do not consider the ones associated directly to $\Lambda$ but we introduce a 40-dimensional even unimodular lattice constructed from $\Lambda$.

3. A certain 40-dimensional even unimodular lattice. An even unimodular lattice $\Gamma$ is obtained by gluing the lattice $\Lambda_{0}$ ( $\Lambda$ is assumed to be odd, unimodular, of minimum 4 and dimension $n<39$ ) with the root lattice $D_{40-n}$ (if $n=39$ one should take instead $\sqrt{2} A_{1}$ ). Then the discriminant groups $\Lambda_{0}^{*} / \Lambda_{0} \cong D_{40-n}^{*} / D_{40-n}$ are isomorphic to $\mathbb{Z} / 4 \mathbb{Z}$ if $n$ is odd and to $\mathbb{Z} / 2 \mathbb{Z} \times \mathbb{Z} / 2 \mathbb{Z}$ if $n$ is even. In order to write down this isomorphism explicitly, we again denote by $s$ and $x$ some fixed minimal vectors of $S$ and $\Lambda \backslash \Lambda_{0}$ respectively. Let $\phi:\left(\Lambda_{0}\right)^{*} / \Lambda_{0} \rightarrow\left(D_{40-n}\right)^{*} / D_{40-n}$ be the isomorphism defined by $\phi(s)=(1 / 2, \ldots, 1 / 2)$ and $\phi(x)=(1,0, \ldots, 0)$. Then, for all 
$u \in\left(\Lambda_{0}\right)^{*} / \Lambda_{0}, \phi(u) \cdot \phi(u) \equiv-u \cdot u \bmod 2 \mathbb{Z}$ (because $2 s$ is a characteristic vector for $\Lambda$, we have $4 s \cdot s \equiv n \bmod 8)$. Let

$$
\Gamma:=\left\{(u, \phi(u)) \in\left(\Lambda_{0}\right)^{*} \perp\left(D_{40-n}\right)^{*}\right\} .
$$

Clearly, the lattice $\Gamma$ is an even unimodular lattice of dimension 40. Its root lattice is $D_{40-n}$. Its vectors of norm 4 are of three types: the ones from $D_{40-n}$, the ones from $\Lambda_{0}$, and the pairs $\left(s^{\prime}, t\right)$ with $s^{\prime} \in S$ of minimal norm and $t \in \phi\left(s^{\prime}\right)$, of minimal norm $10-n / 4$. The number of such vectors $t$ is $2^{39-n}$.

4. Theta series with spherical coefficients. In this section we recall some basic facts about harmonic polynomials and theta series with spherical coefficients associated to even unimodular lattices. We refer to [1], [2, Chapter 18], [4], [7], [8]. The harmonic polynomials are the polynomials in $\mathbb{R}\left[x_{1}, \ldots, x_{n}\right]$ which are homogeneous and satisfy $L P=0$ where $L=\sum \partial^{2} / \partial x_{i}^{2}$ is the Laplace operator. It is a classical result that the formula

$$
P_{k, \alpha}(x)=G_{k}\left((x \cdot \alpha),((x \cdot x)(\alpha \cdot \alpha))^{1 / 2}\right),
$$

where $G_{k}(t, 1)$ is the Gegenbauer polynomial of degree $k$ and parameter $n / 2-1$, defines a harmonic polynomial. For example

$$
P_{2, \alpha}(x)=(x \cdot \alpha)^{2}-\frac{1}{n}(\alpha \cdot \alpha)(x \cdot x) .
$$

We shall also need the polynomial $P_{6, \alpha}(x)$ relative to dimension 40 :

$$
\begin{aligned}
P_{6, \alpha}(x)= & (x \cdot \alpha)^{6}-\frac{5}{16}(x \cdot \alpha)^{4}(\alpha \cdot \alpha)(x \cdot x) \\
& +\frac{15}{736}(x \cdot \alpha)^{2}(\alpha \cdot \alpha)^{2}(x \cdot x)^{2}-\frac{5}{32384}(\alpha \cdot \alpha)^{3}(x \cdot x)^{3}
\end{aligned}
$$

A classical result due to Hecke asserts that, if $P$ is a harmonic polynomial of degree $k$ and if $\Gamma$ is an even unimodular lattice, then

$$
\theta_{\Gamma, P}(\tau):=\sum_{x \in \Lambda} P(x) q^{(x \cdot x)}
$$

defines a modular form for the full modular group $\mathrm{SL}(2, \mathbb{Z})$ of weight $n / 2+k$. The algebra of modular forms for the full modular group is a polynomial algebra in the elements $E_{4}, E_{6}$ of respective weights 4 and 6 :

$$
\begin{aligned}
& E_{4}(\tau)=1+240 \sum_{r=1}^{\infty} \sigma_{3}(r) q^{2 r}=1+240 q^{2}+240 \cdot 9 q^{4}+\ldots \\
& E_{6}(\tau)=1-504 \sum_{r=1}^{\infty} \sigma_{5}(r) q^{2 r}=1-504 q^{2}-504 \cdot 33 q^{4}+\ldots
\end{aligned}
$$


The cusp form of lowest weight is the weight 12 form

$$
\Delta_{12}=\left(E_{4}^{3}-E_{6}^{2}\right) / 1728=q^{2} \prod_{r=1}^{\infty}\left(1-q^{r}\right)^{24} .
$$

5. Equations. In this section we derive some equations satisfied by the numbers $p_{i}(s)$ and $m_{i}(s)$. We take the notations of Section 2 and consider the lattice $\Gamma$ constructed in Section 3. We introduce the additional notations:

- $l_{4}$ is the number of norm 4 vectors in $\Lambda$,

- $d_{2}, d_{4}$ are the numbers of norm 2 , respectively norm 4 vectors in $D_{40-n}$.

Let $\alpha$ belong to the vector space spaced by $\Lambda_{0}$, and let $f_{k}:=\theta_{\Gamma, P_{k, \alpha}}$ be defined in the previous section. For all $x \in \Gamma_{2}, x \cdot \alpha=0$ so the coefficient of $q^{2}$ in $f_{k}$ is $\sum_{x \in \Gamma_{2}} P_{k, \alpha}(x)=G_{k}\left(0,2^{1 / 2}(\alpha \cdot \alpha)^{1 / 2}\right) d_{2}$.

Taking account of the three types of norm 4 vectors in $\Gamma$, the coefficient of $q^{4}$ is

$$
\begin{aligned}
\sum_{x \in \Gamma_{4}} P_{k, \alpha}(x)= & G_{k}\left(0,2(\alpha \cdot \alpha)^{1 / 2}\right) d_{4}+\sum_{x \in \Lambda_{4}} G_{k}\left((x \cdot \alpha), 2(\alpha \cdot \alpha)^{1 / 2}\right) \\
& +2^{39-n} \sum_{s^{\prime} \in S_{m}} G_{k}\left(\left(s^{\prime} \cdot \alpha\right), 2(\alpha \cdot \alpha)^{1 / 2}\right)
\end{aligned}
$$

If $k=2$, the weight of $f_{k}$ is $20+2=22$ so $f_{k}$ is a multiple of $\Delta_{12} E_{4} E_{6}=$ $q^{2}-288 q^{4}+\ldots$; the multiplicity factor is exactly

$$
G_{k}\left(0,2^{1 / 2}(\alpha \cdot \alpha)^{1 / 2}\right) d_{2}=-\frac{2(\alpha \cdot \alpha)}{40} d_{2} .
$$

We now take a minimal vector $\alpha=s$ in $S$; the equality of the coefficients of $q^{4}$ leads, by the expression for $G_{2}$, to the equation

$$
\begin{aligned}
\sum_{x \in \Lambda_{4}}\left((x \cdot s)^{2}-4 m / 40\right)+2^{39-n} \sum_{s^{\prime} \in S_{m}}\left(\left(s^{\prime} \cdot s\right)^{2}\right. & -4 m / 40) \\
& =\frac{576 m}{40} d_{2}+\frac{4 m}{40} d_{4},
\end{aligned}
$$

which leads to the following equation for the $p_{i}(s)$ and $m_{i}(s)$ :

$$
\begin{aligned}
\sum_{i} i^{2} p_{i}(s)+2^{39-n} \sum_{i} i^{2} m_{i}(s) & \\
= & \frac{72 m}{5} d_{2}+\frac{m}{10} d_{4}-2^{40-n} m^{2}+\frac{m}{10}\left(l_{4}+2^{39-n} s_{m}\right) .
\end{aligned}
$$

If $k=4$, we do not get a similar equation because the weight is 24 and the corresponding space of cusp forms is two-dimensional spanned by $\Delta_{12} E_{6}^{2}$ and $\Delta_{12}^{2}$. If $k=6$ the situation is better because the only cusp form of weight 26 is, up to a multiplicative factor, $\Delta_{12} E_{4}^{2} E_{6}=q^{2}-48 q^{4}+\ldots$ We 
compute this factor from the coefficient of $q^{2}$. We get

$$
\begin{aligned}
\sum_{x \in \Lambda_{4}} G_{6}\left((x \cdot \alpha), 2(\alpha \cdot \alpha)^{1 / 2}\right) & +2^{39-n} \sum_{s^{\prime} \in S_{m}} G_{6}\left(\left(s^{\prime} \cdot \alpha\right), 2(\alpha \cdot \alpha)^{1 / 2}\right) \\
& =-\frac{5}{32384}\left(-2^{3} \cdot 48 d_{2}-4^{3} d_{4}\right)(\alpha \cdot \alpha)^{3} .
\end{aligned}
$$

This equation leads to an equation in the $p_{i}(s), m_{i}(s)$ when $\alpha=s$ belongs to $S_{m}$ :

$$
\begin{aligned}
\sum_{i} G_{6}\left(i, 2 m^{1 / 2}\right) p_{i}(s)+2^{39-n} \sum_{i} G_{6}\left(i, 2 m^{1 / 2}\right) m_{i}(s) \\
=-\frac{5}{32384}\left(-2^{3} \cdot 48 d_{2}-4^{3} d_{4}\right) m^{3}-2^{40-n} G_{6}\left(m, 2 m^{1 / 2}\right) .
\end{aligned}
$$

The equation (19) holds for all $\alpha$ in the $n$-dimensional space spanned by $\Lambda$, so we can also view it as a polynomial identity in the coordinates of $\alpha$ and apply the Laplace operator corresponding to this space. This leads to a degree 4 identity.

Let $L_{n}$ denote the Laplace operator in the $n$ variables of $\alpha$. We use the following identity, valid for all $y \in \mathbb{R} \Lambda([7])$ :

$$
\begin{aligned}
& L_{n}\left((\alpha \cdot \alpha)^{l}(\alpha \cdot y)^{k}\right) \\
= & 2 l(2 l+2 k+n-2)(\alpha \cdot \alpha)^{l-1}(\alpha \cdot y)^{k}+k(k-1)(y \cdot y)(\alpha \cdot \alpha)^{l}(\alpha \cdot y)^{k-2,},
\end{aligned}
$$

and obtain an expression for $L_{n}\left(G_{6}\left((y \cdot \alpha), 2(\alpha \cdot \alpha)^{1 / 2}\right)\right)$ :

$$
\begin{aligned}
L_{n}\left(G_{6}\left((y \cdot \alpha), 2(\alpha \cdot \alpha)^{1 / 2}\right)\right) & \\
= & \left(30(y \cdot y)-\frac{5}{2}(8+n)\right)(y \cdot \alpha)^{4} \\
& +\left(-15(y \cdot y)+\frac{30}{23}(6+n)\right)(\alpha \cdot \alpha)(y \cdot \alpha)^{2} \\
& +\left(\frac{15}{23}(y \cdot y)-\frac{15}{253}(4+n)\right)(\alpha \cdot \alpha)^{2} .
\end{aligned}
$$

Then we again take $\alpha=s$ and find a fifth equation for the $p_{i}(s), m_{i}(s)$.

\section{Proof of Theorem 1}

6.1. Dimension 34. The system of five equations found in Section 5 on the unknowns $p_{0}(s), p_{1}(s), p_{2}(s), m_{0}(s), m_{1 / 2}(s)$ has a unique solution $p_{0}(s)=42780, p_{1}(s)=17300, p_{2}(s)=100, m_{0}(s)=102, m_{1 / 2}(s)=100$.

The quotient $\left(\Lambda_{0}\right)^{*} / \Lambda_{0}$ is isomorphic to $\mathbb{Z} / 2 \mathbb{Z} \times \mathbb{Z} / 2 \mathbb{Z}$. The three subgroups of order 2 define three lattices, one is $\Lambda$ and the remaining two are dual to each other; we denote them by $L$ and $L^{*}$. Clearly two short vectors $s, s^{\prime} \in S_{m}$ are both in $L$ or $L^{*}$ if and only if $s \cdot s^{\prime}= \pm 1 / 2$. 
Let $s \in S_{m}$ be a fixed vector. Let

$$
X:=\{s\} \cup\left\{s^{\prime} \in S_{m} \mid s \cdot s^{\prime}=1 / 2\right\} .
$$

From the computation of $m_{1 / 2}(s)$ we know that the cardinality of $X$ is 51. Let $G$ be the Gram matrix of this set, where $s$ is chosen to be the first of the vectors in $X$.

LEMMA $1 . G^{2}=\frac{15}{2} G$.

Proof. We compute $G^{2}: G^{2}\left[s^{\prime}, s^{\prime \prime}\right]=\sum_{x \in X}\left(s^{\prime} \cdot x\right)\left(x \cdot s^{\prime \prime}\right)$. The vectors of $S_{m}$ are either in $\pm X$ or are perpendicular to $X$, so

$$
G^{2}\left[s^{\prime}, s^{\prime \prime}\right]=\frac{1}{2} \sum_{x \in S_{m}}\left(s^{\prime} \cdot x\right)\left(x \cdot s^{\prime \prime}\right) .
$$

From the values found for $m_{0}(s)$ and $m_{1 / 2}(s)$, one can check that the set $S_{m}$ is a 2-design because $\sum_{s^{\prime}, s^{\prime \prime} \in S_{m}}\left(s^{\prime} \cdot s^{\prime \prime}\right)^{2}=m^{2} s_{m}^{2} / n$ (see [7, Theorem 8.1]). Hence, for all $\alpha$,

$$
\sum_{x \in S_{m}}(\alpha \cdot x)^{2}=\frac{m s_{m}}{n}(\alpha \cdot \alpha)=15(\alpha \cdot \alpha) .
$$

Applied to $\alpha+\beta$, this identity leads to $\sum_{x \in S_{m}}(\alpha \cdot x)(x \cdot \beta)=15(\alpha \cdot \beta)$ for all $\alpha, \beta$, and, when $\alpha=s^{\prime}, \beta=s^{\prime \prime}$, to the statement $G^{2}=\frac{15}{2} G$.

We now consider the graph with vertices $X \backslash\{s\}$ and edges the pairs $\left(s^{\prime}, s^{\prime \prime}\right)$ with $\left(s^{\prime} \cdot s^{\prime \prime}\right)=-1 / 2$. This graph is regular with valency 22 as can be checked from the computation of the coefficient $\left(s, s^{\prime}\right)$ in the identity $G^{2}=\frac{15}{2} G$. If $A$ is the incidence matrix of this graph and if $A^{\prime}$ is the matrix obtained from $A$ by adding a first row of zeros and a first column of zeros, we have

$$
G=2 I_{51}+\frac{1}{2} J_{51}-A^{\prime}
$$

where $I_{p}$ denotes the identity matrix of size $p$, and $J_{p}$ denotes the $p \times p$ matrix with all entries 1 . Putting this in the equation $G^{2}=\frac{15}{2} G$ and taking account of the identity $A J_{50}=J_{50} A=22 J_{50}$, we get

$$
A^{2}-\frac{7}{2} A-11 J_{50}-11 I_{50}=0 .
$$

Of course, this last identity is not possible for a matrix $A$ with entries 0 or 1 so we deduce the non-existence of the lattice $\Lambda$.

6.2. Dimension 35. The system of five equations found in Section 5 on the unknowns $p_{0}(s), p_{1}(s), p_{2}(s), m_{1 / 4}(s), m_{3 / 4}(s)$ has a unique solution $p_{0}(s)=35289, p_{1}(s)=15642, p_{2}(s)=99, m_{1 / 4}(s)=319, m_{3 / 4}(s)=99$. But these numbers should be even so the lattice $\Lambda$ does not exist. 


\section{References}

[1] C. Bachoc and B. Venkov, Modular forms, lattices and spherical designs, in: Réseaux euclidiens, "designs" sphériques et groupes, J. Martinet (éd.), Enseign. Math. Monographie 37, Genève, to appear.

[2] J. Conway and N. J. A. Sloane, Sphere Packings, Lattices and Groups, Springer, 1988.

[3] —, —, A note on optimal unimodular lattices, J. Number Theory 72 (1998), 357-362.

[4] W. Ebeling, Lattices and Codes, Vieweg, 1994.

[5] G. Nebe and N. J. A. Sloane, A catalogue of lattices, http://www.research.att.com/ $\sim$ njas/lattices.

[6] E. M. Rains and N. J. A. Sloane, The shadow theory of modular and unimodular lattices, J. Number Theory 73 (1998), 359-389.

[7] B. Venkov, Réseaux et designs sphériques, in: Réseaux euclidiens, "designs" sphériques et groupes, J. Martinet (éd.), Enseign. Math. Monographie 37, Genève, to appear.

[8] -, Even unimodular extremal lattices, Proc. Steklov Inst. Math. 165 (1984), 47-52.

Laboratoire A2X

Université Bordeaux I

351, cours de la Libération

33405 Talence, France

E-mail: bachoc@math.u-bordeaux.fr

St Petersburg Branch of the Steklov Institut of Mathematics

Fontanaka 27

191011 St Petersburg, Russia

E-mail: bvenkov@pdmi.ras.ru
Abteilung Reine Mathematik

Universität Ulm

89069 Ulm, Germany

E-mail: nebe@mathematik.uni-ulm.de

Received on 27.12.2000 